\title{
Regulation and Biological Significance of Formation of Osteoclasts and Foreign Body Giant Cells in an Extraskeletal Implantation Model
}

\author{
Gazi Jased Ahmed ${ }^{1,2, *}$, Eri Tatsukawa ${ }^{2, *}$, Kota Morishita ${ }^{1,2}$, Yasuaki Shibata ${ }^{2, \dagger}$, \\ Fumio Suehiro ${ }^{3}$, Masanobu Kamitakahara ${ }^{4}$, Taishi Yokoi ${ }^{4, *}$, Takehiko Koji ${ }^{5}$, \\ Masahiro Umeda ${ }^{1}$, Masahiro Nishimura ${ }^{3}$ and Tohru Ikeda ${ }^{2}$ \\ ${ }^{I}$ Departments of Clinical Oncology, Nagasaki University Graduate School of Biomedical Sciences, ${ }^{2}$ Department of Oral \\ Pathology and Bone Metabolism, Nagasaki University Graduate School of Biomedical Sciences, 1-7-1 Sakamoto, Nagasaki \\ 852-8588, Japan, ${ }^{3}$ Department of Prosthodontics, Kagoshima University Graduate School, 8-35-1 Sakuragaoka, Kagoshima \\ 890-8544, Japan, ${ }^{4}$ Graduate School of Environmental Studies, Tohoku University, 6-6-20, Aramaki, Aoba-ku, Sendai, Miyagi \\ 980-8579, Japan and ${ }^{5}$ Department of Histology and Cell Biology, Nagasaki University Graduate School of Biomedical \\ Sciences, 1-12-4 Sakamoto, Nagasaki 852-8523, Japan
}

Received March 8, 2016; accepted May 31, 2016; published online June 16, 2016

\begin{abstract}
The implantation of biomaterials induces a granulomatous reaction accompanied by foreign body giant cells (FBGCs). The characterization of multinucleated giant cells (MNGCs) around bone substitutes implanted in bone defects is more complicated because of healing with bone admixed with residual bone substitutes and their hybrid, and the appearance of two kinds of MNGCs, osteoclasts and FBGCs. Furthermore, the clinical significance of osteoclasts and FBGCs in the healing of implanted regions remains unclear. The aim of the present study was to characterize MNGCs around bone substitutes using an extraskeletal implantation model and evaluate the clinical significance of osteoclasts and FBGCs. Betatricalcium phosphate ( $\beta$-TCP) granules were implanted into rat subcutaneous tissue with or without bone marrow mesenchymal cells (BMMCs), which include osteogenic progenitor cells. We also compared the biological significance of plasma and purified fibrin, which were used as binders for implants. Twelve weeks after implantation, osteogenesis was only detected in specimens implanted with BMMCs. The expression of two typical osteoclast markers, tartrate-resistant acid phosphatase (TRAP) and cathepsin-K (CTSK), was analyzed, and TRAP-positive and CTSK-positive osteoclasts were only detected beside bone. In contrast, most of the MNGCs in specimens without the implantation of BMMCs were FBGCs that were negative for TRAP, whereas the degradation of $\beta$-TCP was detected. In the region implanted with $\beta$-TCP granules with plasma, FBGCs tested positive for CTSK, and when $\beta$-TCP granules were implanted with purified fibrin, FBGCs tested negative for CTSK. These results showed that osteogenesis was essential to osteoclastogenesis, two kinds of FBGCs, CTSK-positive and CTSK-negative, were induced, and the expression of CTSK was plasma-dependent. In addition, the implantation of BMMCs was suggested to contribute to osteogenesis and the replacement of implanted $\beta$-TCP granules to bone.
\end{abstract}

Key words: osteoclast, foreign body giant cell, beta-tricalcium phosphate, cathepsin $\mathrm{K}$, macrophage

\footnotetext{
*These authors contributed equally to this work.

Correspondence to: Tohru Ikeda, Department of Oral Pathology and Bone Metabolism, Nagasaki University Graduate School of Biomedical Sciences, 1-7-1 Sakamoto, Nagasaki 852-8588, Japan.

E-mail: tohrupth@nagasaki-u.ac.jp

Present address: Department of Histology and Cell Biology, Nagasaki University Graduate School of Biomedical Sciences, 1-12-4 Sakamoto, Nagasaki 852-8523, Japan

Present address: Materials Research and Development Laboratory, Japan Fine Ceramics Center, 2-4-1 Mutsuno, Atsuta-ku, Nagoya 456-8587, Japan
}

\section{Introduction}

Foreign body reactions with the formation of granulomas occur in every site of implantation following an acute inflammatory phase $[1,15]$. Granulomas composed of epithelioid cells and foreign body giant cells (FBGCs) make contact with the implant surface. In the cases of bone substitutes and dental implants, granulomas are replaced with bone following osteogenesis. When implanted into 
bone defects, non-biodegradable bone substitutes such as hydroxyapatite (HA) are expected to be involved in regenerative bone tissue and form a hybrid [25]. When biodegradable bone substitutes are implanted into bone defects, the degradation of bone substitutes and osteogenesis occur simultaneously, and FBGCs and/or osteoclasts may contribute to the replacement of bone substitutes with bone. We previously reported that multinucleated giant cells (MNGCs) with tartrate-resistant acid phosphatase (TRAP) activity around bone substitutes may be regarded as osteoclasts rather than FBGCs at least 4 weeks after implantation because of their involvement in normal bone metabolism to form trabecular structures $[6,7,9]$. These findings indicate that foreign body reactions are exchanged for bone metabolic reactions after involvement into bone. However, FBGCs may resorb bone substitutes in collaboration with osteoclasts during the process of involvement into bone. Furthermore, multiple kinds of MNGCs may play roles in this very complex in vivo condition. The granulomatous reaction is considered to protect tissue from foreign bodies by isolation; however, it sometimes interferes with the regeneration of wounds. In contrast to FBGCs, osteoclasts are physiologically essential for bone metabolism, and without the pathological stimulation of osteoclast activity, which is typically induced by inflammation, osteoclasts may contribute to the regeneration of bone defects implanted with bone substitutes. However, the clinical significance of osteoclasts and FBGCs in a region implanted with bone substitutes remains unclear.

The biological mechanisms by which FBGCs form have been studied in vitro, and FBGCs were previously reported to be generated from macrophages treated with interleukin-4 (IL-4) or IL-13 [4, 19, 21]. FBGCs generated in vitro were recently suggested to exhibit TRAP activity [12]. In addition, MNGCs in human granulomatous lesions including tuberculosis may be positive for TRAP and cathepsin K (CTSK) [27]. TRAP-positive FBGCs have been suggested to exhibit little or no bone-resorbing activity [12]. However, these cells may share biological similarities with osteoclasts, which are induced from macrophages stimulated with macrophage colony-stimulating factor (M-CSF) and receptor activator of nuclear factorkappa B ligand (RANKL) [16, 33]. These findings also indicate that there are multiple kinds of FBGCs because these cells are typically negative for TRAP.

We have been investigating biological responses to HA and $\beta$-tricalcium phosphate ( $\beta$-TCP) with different microstructures. Our findings strongly suggest that microstructures influence biological responses when implanted into bone defects, and may, at least partly, be involved in affinity for serum proteins [10]. HA composed of rodshaped particles, which is synthesized by applied hydrothermal methods, was found to have weak biodegradability and a potent osteoclast-homing capacity $[7,9,26]$. BetaTCP composed of rod-shaped particles exhibited stable biodegradability and induced cancellous bone with a phys- iological trabecular structure, even in ovariectomized rats [29]. In contrast, the implantation of $\beta$-TCP synthesized by normal sintering had higher biodegradability, and $\beta$-TCP granules were degraded by surrounding TRAP-positive MNGCs in a rabbit model [25]. In the present study, we implanted $\beta$-TCP granules synthesized by normal sintering into rat subcutaneous tissue with or without bone marrow mesenchymal cells (BMMCs), which include osteogenic progenitor cells $[18,24]$. When implanted into extraskeletal tissue, $\beta$-TCP is expected to induce FBGCs rather than osteoclasts because $\beta$-TCP does not accompany osteoblasts, which induce osteoclasts by the expression of RANKL. However, the further implantation of BMMCs is expected to induce bone, and osteoclasts may appear in addition to FBGCs. FBGCs near bone interfaces are suggested to differ from those formed in soft tissue [1], and this subcutaneous implantation model suits the characterization of MNGCs not only by differentiation markers, but also by the degradation of $\beta$-TCP. The results of the present study may provide an insight into biological differences between osteoclasts and FBGCs, and the clinical significance of these MNGCs in the healing of regions implanted with bone substitutes.

\section{Materials and Methods}

\section{Preparation of $\beta$-TCP granules and discs}

Beta-TCP granules were synthesized using the following method. An aqueous slurry was prepared by mixing $10 \mathrm{~g}$ of $\beta$-TCP $\left(\mathrm{Ca}_{3}\left(\mathrm{PO}_{4}\right)_{2}\right.$, Taihei Chemical, Osaka, Japan) and $50 \mathrm{~g}$ of 10 mass\% gelatin (Wako Pure Chemical, Osaka, Japan) aqueous solution. The slurry was added to $1.8 \mathrm{dm}^{3}$ of vegetable oil under stirring at approximately $200 \mathrm{rpm}$ at $5^{\circ} \mathrm{C}$ for $1 \mathrm{hr}$. The resultant granules were collected, washed with ethanol, and dried in a refrigerator at $4^{\circ} \mathrm{C}$. They were then heated at $1000^{\circ} \mathrm{C}$ for $2 \mathrm{hr}$ to remove residual gelatin and sinter. Granules of approximately $500-600 \mu \mathrm{m}$ in diameter were collected using sieves. In order to prepare $\beta$ TCP disc samples, $0.3 \mathrm{~g}$ of $\beta$-TCP was inserted into a mold that was $14 \mathrm{~mm}$ in diameter and pressed at $200 \mathrm{MPa}$. The samples were heated at $900^{\circ} \mathrm{C}$ for $3 \mathrm{hr}$.

\section{Preparation of BMMCs}

Three- to 4-week-old male F344 rats (CLEA Japan Inc., Shizuoka, Japan) were purchased for these experiments, euthanized by the inhalation of carbon dioxide, and the tibiae and femora were removed. Both ends of these bones were cut and bone marrow was flushed out using a 5 -ml syringe equipped with an 18-gauge needle and filled with growth medium, composed of minimum essential medium alpha (Sigma-Aldrich, St. Louis, MO) supplemented with $10 \%$ fetal bovine serum (Sigma-Aldrich) and penicillin-streptomycin-glutamine (Life Technologies, Carlsbad, CA). Bone marrow was dissociated by pipetting and plated on six 82-mm culture dishes (Asahi Techno Glass, Shizuoka, Japan). One week after plating, BMMCs 
were dislodged using trypsin-EDTA (Life Technologies) and re-plated on 10 culture dishes. Two weeks later, BMMCs were dislodged as described and used for implantation.

\section{Implantation}

Forty milligrams of $\beta$-TCP granules were mixed in a $1.5-\mathrm{ml}$ micro-centrifuge tube with $2 \times 10^{6}$ of BMMCs suspended in $600 \mu \mathrm{l}$ of growth medium and placed in a $37^{\circ} \mathrm{C}$ incubator for $3 \mathrm{hr}$. Growth medium was removed and 100 $\mu \mathrm{l}$ of 4-week-old male F344 rat plasma prepared from frozen stock was added. $\beta$-TCP granules were directly soaked in plasma in a $1.5-\mathrm{ml}$ micro-centrifuge tube for implantation without BMMCs. Plasma in each tube was gelled by the addition of $10 \mu \mathrm{l}$ of $3.3 \%$ calcium chloride. In other experiments, $\beta$-TCP granules were soaked in $100 \mu \mathrm{l}$ of Bolheal $^{\circledR}$ (Kaketsuken, Kumamoto, Japan), which was made from human fibrinogen and gelled with thrombin following the manufacturer's protocol. Ten-week-old F344 rats were anesthetized with ketamine and xylazine (intraperitoneal injections at doses of $40 \mathrm{mg} / \mathrm{kg}$ and $3 \mathrm{mg} / \mathrm{kg}$ body weight, respectively). These gels were implanted into the back subcutaneous tissue of the anesthetized F344 rats. The gels were implanted into two portions (left and right) on the back of each rat. Four implantations were performed for each group in these experiments. Twelve weeks after implantation, animals were euthanized as described. Implanted portions were resected and fixed with $4 \%$ paraformaldehyde in $0.1 \mathrm{M}$ phosphate buffer $(\mathrm{pH} 7.2)$ at $4^{\circ} \mathrm{C}$ for $24 \mathrm{hr}$. After fixation, a micro-CT analysis was performed for implants using an R_mCT (Rigaku, Tokyo, Japan). Each implant was then cut in half with a razor, with one being used for undecalcified sections and the other for decalcified sections. Animal rearing and experiments were performed at the Biomedical Research Center, Center for Frontier Life Sciences, Nagasaki University, following the Guidelines for Animal Experimentation of Nagasaki University (Approval no. 1112190960) under specific pathogenfree conditions.

\section{Histological procedures}

Regarding undecalcified sections, fixed specimens were dehydrated and embedded in 2-hydroxyethyl methacrylate/methyl methacrylate/2-hydroxyethyl acrylate mixed resin (Wako Pure Chemical). Three-micrometer-thick sections were made and stained with Giemsa's azur eosin methylene blue solution (Merck, Darmstadt, Germany) or histochemically analyzed to detect TRAP activity using naphthol AS-MX phosphate (Sigma-Aldrich) as a substrate, fast red RC salt (Sigma-Aldrich) as a coupler, and $0.05 \mathrm{M}$ sodium tartrate (Wako Pure Chemical) in $0.05 \mathrm{M}$ acetic acid buffer ( $\mathrm{pH} 5)$. Regarding decalcified sections, fixed specimens were decalcified in $0.5 \mathrm{M}$ ethylenediamine tetraacetic acid (EDTA) solution at $4{ }^{\circ} \mathrm{C}$ with gentle agitation. The decalcified samples were post-fixed with the fixative, dehydrated, and embedded in paraffin. Three-micrometer- thick sections were made and used for further immunohistochemical analyses. The decalcified sections were autoclaved at $121^{\circ} \mathrm{C}$ for $15 \mathrm{~min}$ in $10 \mathrm{mM}$ citrate buffer (DAKO, Carpinteria, CA) and incubated with a CTSK rabbit anti-rat polyclonal antibody (LifeSpan BioSciences, Seattle, WA) at $4^{\circ} \mathrm{C}$ overnight. Immunohistochemical reactions were performed using the EnVision ${ }^{+}$system (DAKO) following the manufacturer's protocol. Sections were counterstained with hematoxylin. Cell numbers were quantitated in 3 optical fields at $\times 100$ magnification. Data were statistically evaluated using the $t$-test and a $P$ value $<0.05$ was considered significant.

\section{PCR analysis}

Five-week-old ddY mice (Japan SLC) were euthanized by cervical dislocation, tibiae and femora were removed, and bone marrow macrophages were prepared, as reported previously [8], in the growth medium described above further supplemented with $30 \mathrm{ng} / \mathrm{ml}$ of M-CSF (Sigma-Aldrich). Synthesized $\beta$-TCP discs were inserted into 24-well wells and $5 \times 10^{4}$ macrophages were plated on ceramic discs and cultured. As a control, macrophages were plated on wells without $\beta$-TCP discs. After 6 days, medium was removed and cells were lysed with TRIzol ${ }^{\circledR}$ reagents (Life Technologies) to extract total RNA. After a DNase treatment, cDNA was synthesized from total RNA with ReverTra Ace (Toyobo, Osaka, Japan). Real-time PCR was performed using Premix Ex Taq (Perfect Real Time) and Thermal Cycler Dice Real Time System Lite (Takara, Shiga, Japan) with the following primers: glyceraldehyde-3phosphate dehydrogenase (GPDH): Mm99999915_g1 TRAP: Mm00475698_m1, CTSK: Mm00484039_m1, NFATc1: Mm00479445_m1, carbonic anhydrase 2 (Car2): Mm00501576_m1 (all from Applied BioSystems, Waltham, MA). The expression levels of each mRNA were normalized to the relative quantity of GPDH mRNA expressed in each sample. Data were statistically evaluated using the $t$-test with data from 3 identical experiments, and a $P$ value $<0.05$ was considered significant.

\section{Results}

\section{Nature of synthesized ceramics}

X-ray diffractometry showed that no phase other than $\beta$-TCP was detected in the granules and discs (Fig. 1). The representative surface structures of $\beta$-TCP granules and discs are shown in Figure 2. Synthesized granules and discs were composed of similar globular-shaped particles, which fused with one another.

\section{Effects of BMMC implantation on the formation of osteoclasts and FBGCs}

Three-dimensional micro-CT images of samples of the implanted portions were shown in Figure 3. Fused radiopaque images, which suggested osteogenesis, were observed in the samples implanted with $\beta$-TCP granules 
with BMMCs (Fig. 3a, in a dotted circle). Although no fused radiopaque images were noted in the samples implanted with $\beta$-TCP without BMMCs, irregular radiopaque images suggestive of the degradation of $\beta$-TCP granules were observed (Fig. 3b, in dotted circles).

Bone tissue was detected in some portions of the specimens implanted with $\beta$-TCP with BMMCs. The marked degradation of $\beta$-TCP granules was apparent in

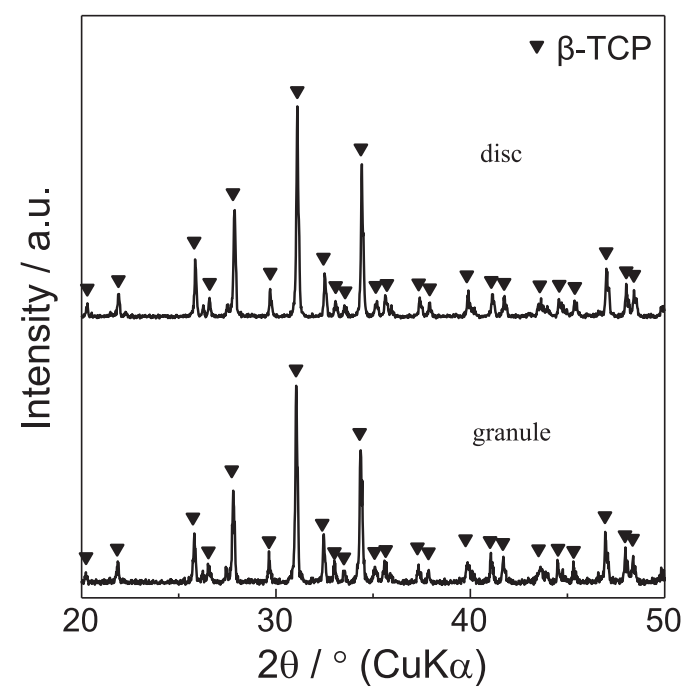

Fig. 1. X-ray diffractometry of $\beta$-TCP granules and discs used in this study. the region with potent osteogenesis, and residual $\beta$-TCP granules were involved in bone (Fig. 4a and 4b). BetaTCP granules that were not completely involved in bone were also strongly degraded (Fig. 4c). The degradation of implanted $\beta$-TCP varied in the region without bone tissue. $\beta$-TCP granules that appeared to be slightly degraded (Fig. $4 \mathrm{~b}$ and $4 \mathrm{c}$, black arrows), fragments of $\beta$-TCP granules caused by dissociation rather than degradation (Fig. $4 \mathrm{~d}$, white arrows), and $\beta$-TCP granules with large deficiencies, which strongly suggested degradation (Fig. 4d, an asterisk), were detected in that region.

One of the important osteoclast markers, TRAP activity was analyzed by a histochemical procedure using serial sections stained with Giemsa solution. TRAP activity was detected in some MNGCs near the bone. Not only MNGCs on the surface of the bone or bone/ $\beta$-TCP hybrid (Fig. 5a, $5 \mathrm{~b}, 5 \mathrm{c}$ and $5 \mathrm{~d}$, arrows), but also MNGCs not directly in contact with the bone or bone/ $\beta$-TCP hybrid were positive for TRAP in specimens showing osteogenesis (Fig. 5a and 5 b, black arrowheads). TRAP-negative MNGCs were also detected near TRAP-positive MNGCs (Fig. 5c and 5d, the white arrowhead). In the region without osteogenesis, almost all MNGCs were negative for TRAP (Fig. 5e and 5f, white arrowheads). Osteogenesis was only observed in two out of 4 samples. The number of TRAP-positive MNGCs in these specimens was counted, and TRAP-positive MNGCs were predominantly detected in specimens with osteogenesis (Fig. 5g). Only MNGCs associated with bone stained strongly for TRAP activity. In contrast, MNGCs in the region without bone showed a negative reaction. Based on
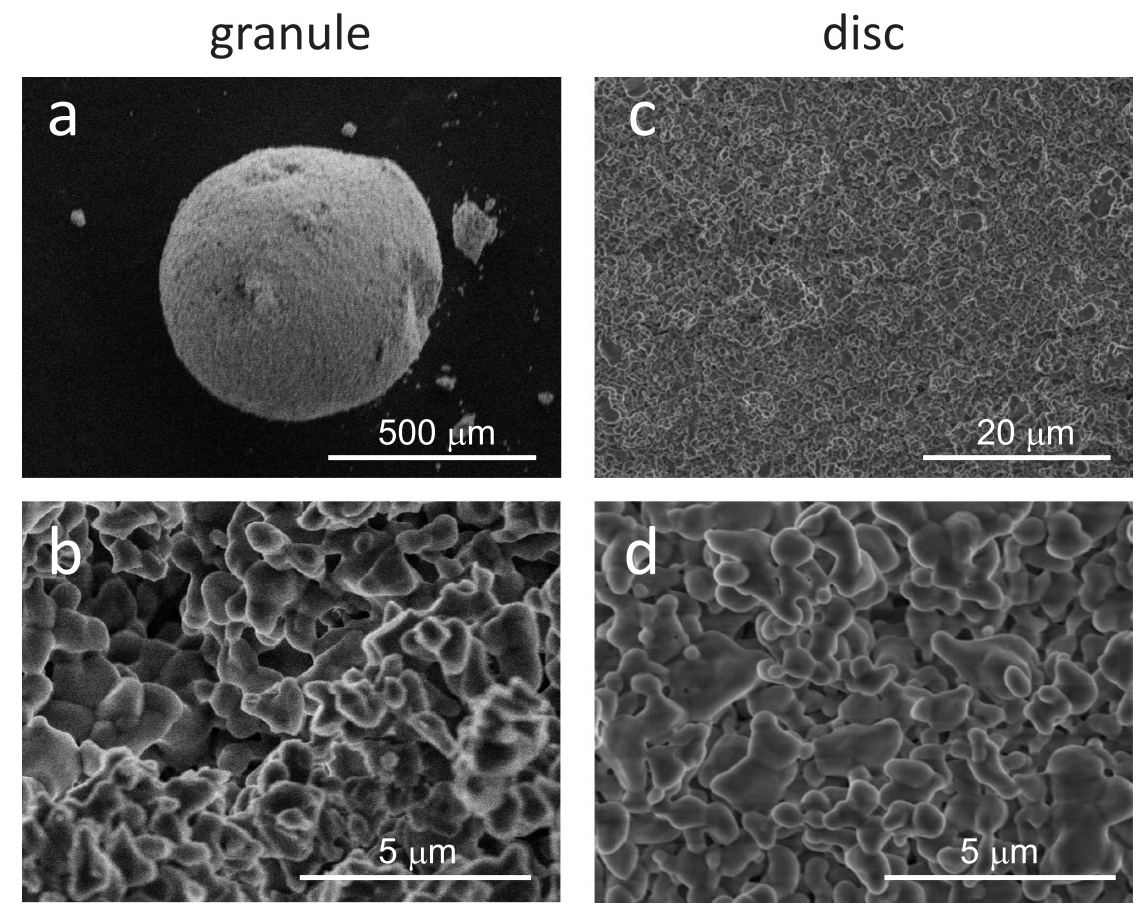

Fig. 2. Scanning electron micrographs of an overview (a) and the microstructure (b) of a $\beta$-TCP granule. A low-power magnification view (c) and the microstructure (d) of a $\beta$-TCP disc. 

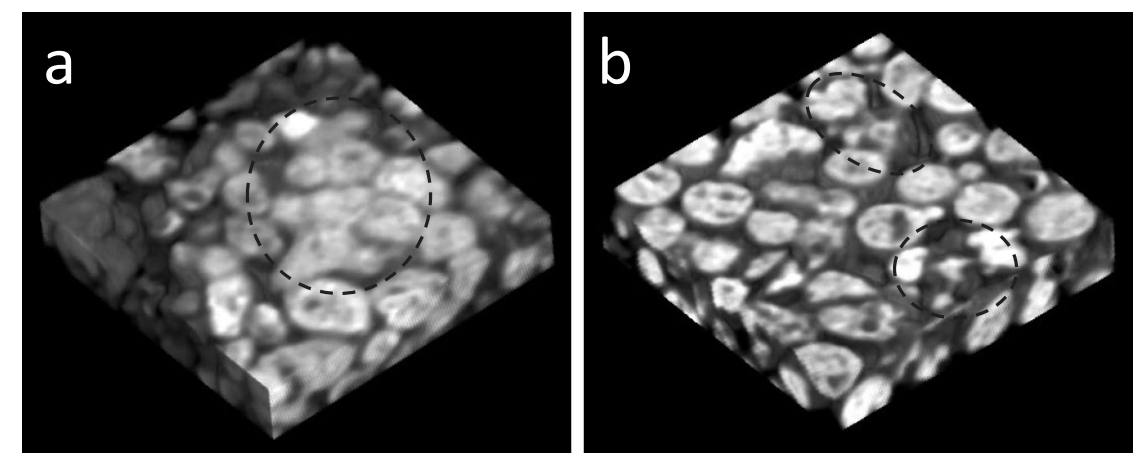

Fig. 3. A three-dimensional image of the region implanted with $\beta$-TCP granules with BMMCs (a) and the region implanted with $\beta$-TCP (b), both of which were bound with plasma. The dotted circle in (a) represents fused radiopaque images suggesting osteogenesis, and the dotted circles in (b) represent the degradation of $\beta$-TCP granules.
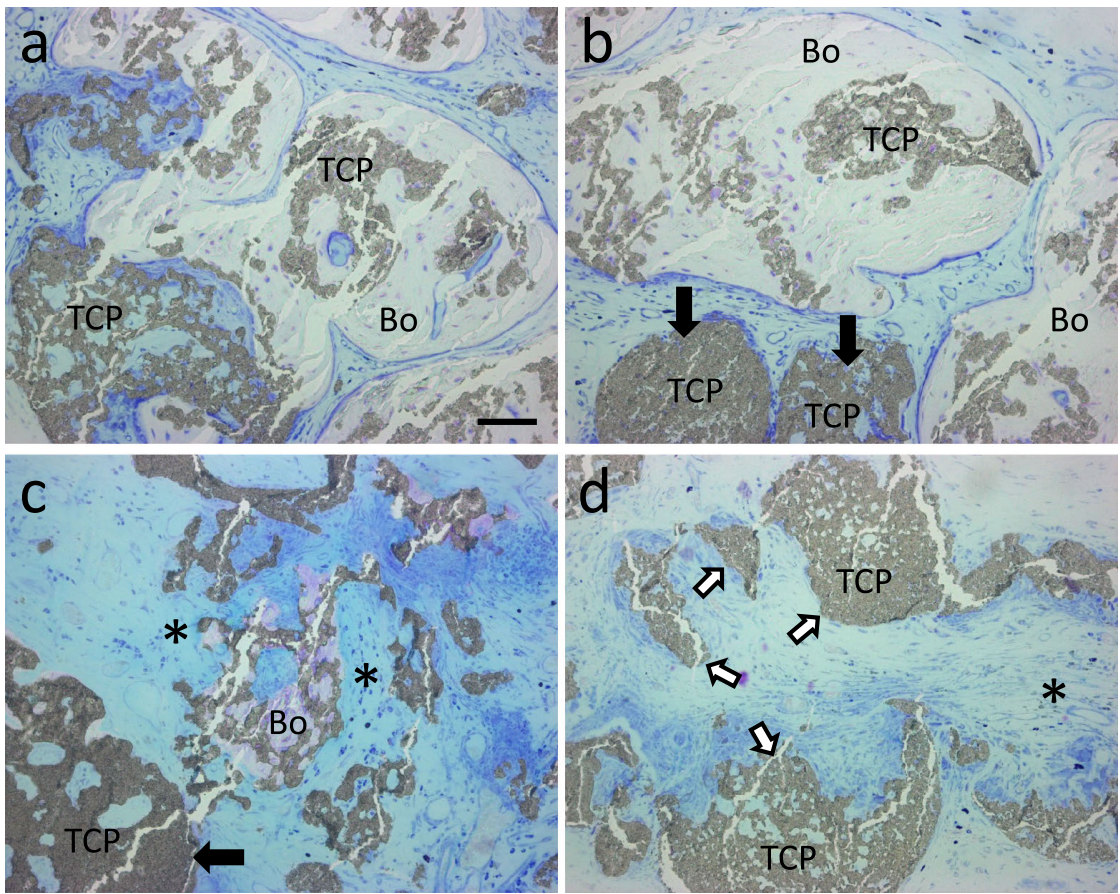

Fig. 4. Histological views of specimens implanted with $\beta$-TCP granules with BMMCs. Sections were stained with Giemsa solution. (a, b) Residual $\beta$ TCP granules (TCP) were surrounded by a large amount of bone (Bo). (c) Partially absorbed $\beta$-TCP fragments were adjacent to a small amount of bone tissue and surrounded by connective tissue $(*)$. Black arrows indicate residual $\beta$-TCP granules that were poorly degraded. (d) The region without osteogenesis, which includes dissociated $\beta$-TCP granules (white arrows) and $\beta$-TCP granules with large deficiencies, was filled with connective tissue $(*)$. Bar $=50 \mu \mathrm{m}$.

these results, TRAP-positive MNGCs (Fig. 6a, b) were defined as osteoclasts and TRAP-negative MNGCs (Fig. 6c, d) as FBGCs.

\section{Effects of plasma components on the expression of CTSK}

We next analyzed the expression of another important osteoclast marker, CTSK using an immunohistochemical procedure. The expression of CTSK in osteoclasts near bone tissue was confirmed (Fig. 7a). In addition to osteoclasts near bone tissue, most FBGCs in the region without osteogenesis were also positive for CTSK (Fig. 7b). In order to analyze the relationship between the induction of CTSK and plasma, which was used as the binder of ceramic granules, specimens implanted with $\beta$-TCP granules that were bound with human fibrin $\left(\right.$ Bolheal $^{\circledR}$ ) were further analyzed. In these specimens, osteogenesis was not detected and FBGCs were present on the surface of $\beta$-TCP granules that were bound with both plasma and fibrin (Fig. $7 \mathrm{c}$ and $7 \mathrm{~d}$, black and white arrowheads), and some FBGCs were suggested to resorb $\beta$-TCP granules and form resorption lacunas (Fig. 7c and 7d, white arrowheads). Most FBGCs in specimens that were bound with fibrin had no or 

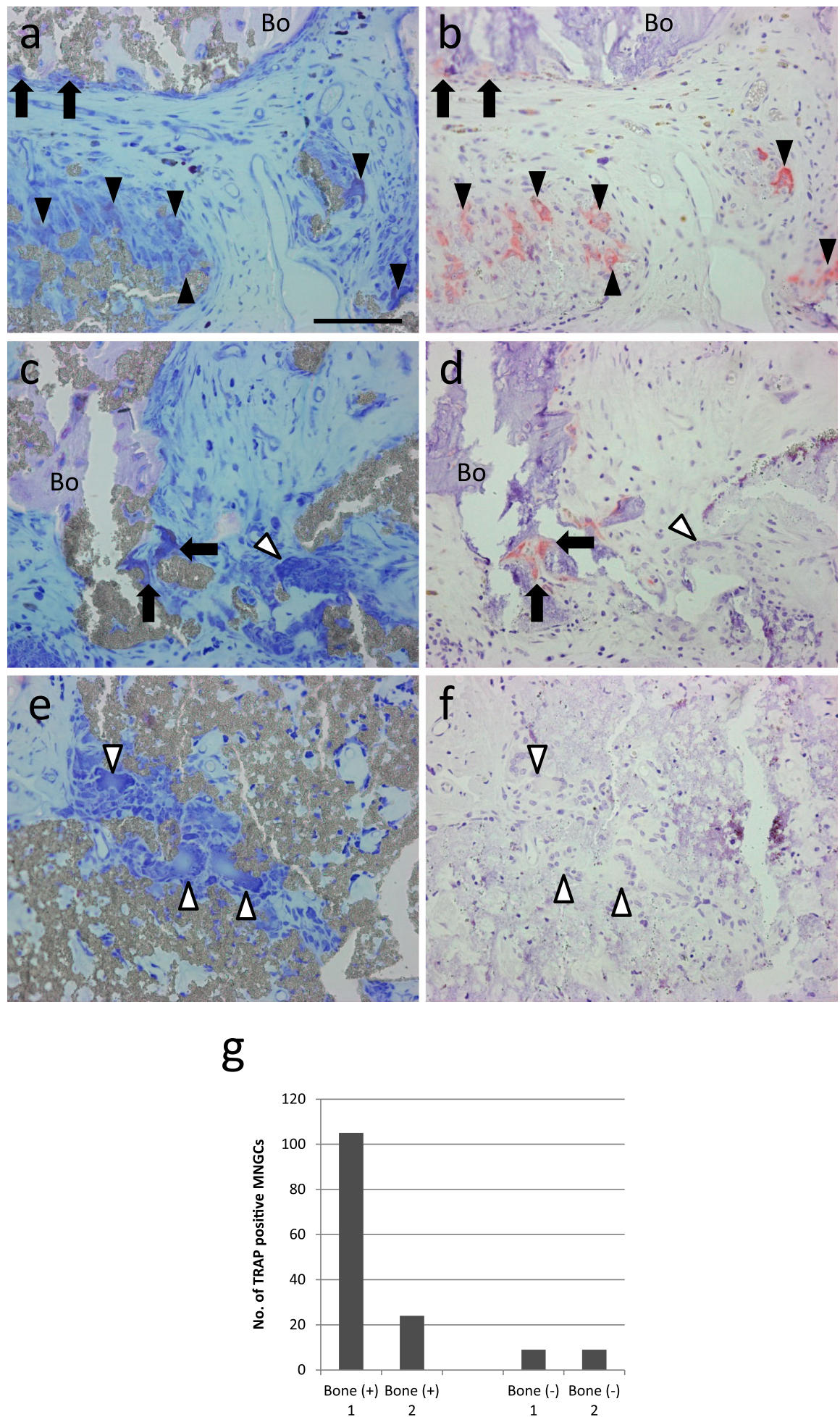

Fig. 5. Histochemical identification of TRAP-positive cells in a specimen implanted with $\beta$-TCP granules with BMMCs. Sets of serial sections (a-f) stained with Giemsa solution (a, c, e) and analyzed for TRAP activity (b, d, f). Arrows indicate TRAP-positive osteoclast-like MNGCs directly in contact with the bone $(\mathrm{Bo})$ or bone/ $\beta$-TCP hybrid, black arrowheads indicate TRAP-positive osteoclast-like MNGCs not directly in contact with the bone or bone/ $\beta$-TCP hybrid, and white arrowheads indicate TRAP-negative MNGCs. Bar $=50 \mu \mathrm{m}$. (g) Quantitation of the number of TRAP-positive MNGCs in 3 optical fields at $\times 100$ magnification of specimens implanted with $\beta$-TCP granules with BMMCs. Two specimens were accompanied by bone formation [Bone $(+)$ ], whereas the other 2 were not [Bone $(-)$ ]. 

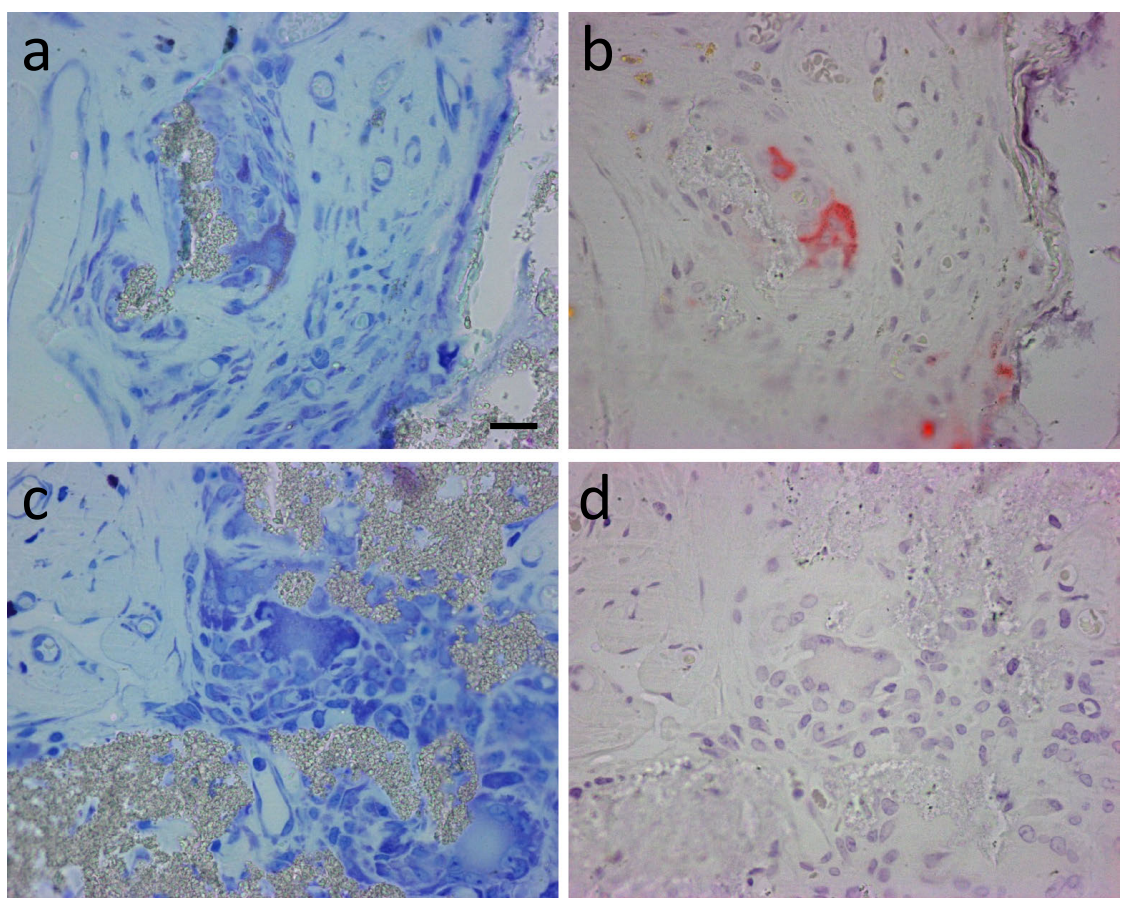

Fig. 6. Characterization of TRAP-positive and TRAP-negative MNGCs. Sets of serial sections (a, b and $\mathbf{c}, \mathbf{d})$ stained with Giemsa solution (a, c) and analyzed for TRAP activity (b, d). (a, b) are larger magnification images of MNGCs in Figure $5(a, b)$, and (c, d) are larger magnification images of MNGCs in Figure $5(\mathrm{e}, \mathrm{f})$. In the specimen implanted with $\beta$-TCP granules with BMMCs, MNGCs near bone exhibit potent TRAP activity (b), whereas MNGCs in the region without bone formation do not possess TRAP activity. Bar=20 $\mu \mathrm{m}$.

a very faint CTSK signal (Fig. 7f, white arrowheads) in contrast to FBGCs in specimens implanted with $\beta$-TCP granules that were bound with plasma (Fig. 6e, black arrowheads). The results of the quantitation of CTSKpositive and -negative FBGCs are shown in Figure $7 \mathrm{~g}$.

\section{Effects of $\beta$-TCP on the expression of osteoclast markers}

$\beta$-TCP may have influenced the expression of osteoclast markers in FBGCs in the implanted region. Therefore, bone marrow macrophages from the ddY mouse, which is widely used in in vitro osteoclastogenesis assays, were seeded on culture dishes with or without the insertion of $\beta$-TCP discs, cultured for 6 days, and the expression of osteoclast markers was then analyzed using real-time PCR. No significant differences were observed in the expression of TRAP, CTSK, or Car2. However, the expression of NFATc1 was significantly weaker in macrophages cultured on $\beta$-TCP discs than in those cultured on plastic culture dishes (Fig. 8).

\section{Discussion}

In the present study, we confirmed that the implantation of BMMCs with $\beta$-TCP granules induced osteogenesis and osteoclastogenesis. Furthermore, the marked degradation of $\beta$-TCP granules in the region strongly suggested that the implantation of BMMCs contributed not only to osteogenesis, but also to the replacement of $\beta$-TCP granules to bone. Hence, our results indicated that osteoclastogenesis rather than the formation of FBGCs is favorable for the regeneration of bone defects implanted with biodegradable bone substitutes if prolonged inflammation, which may destroy regenerated bone and implanted bone substitutes through excess of osteoclast activity, is prevented.

The biological mechanisms underlying osteoclastogenesis have been extensively examined and elucidated in detail since the discovery of the key molecule RANKL, which is expressed in osteoblasts. RANKL and M-CSF induce macrophages to differentiate into mononuclear osteoclast progenitor cells, mononuclear preosteoclasts, and multinuclear osteoclasts $[2,28,31]$. There are several kinds of macrophage-derived MNGCs in addition to osteoclasts. For example, FBGCs appear in foreign body granulomas, Langhans giant cells in tubercle granulomas, and Toutontype giant cells in cholesterol granulomas. In vitro experiments indicated that the treatment of macrophages with IL-4 or IL-13 and M-CSF or GM-CSF induced FBGCs, and IL-4 and IL-13 were identified as key molecules for the induction of FBGCs [1]. Differences in and similarities between non-osteoclastic MNGCs currently remain unclear [3].

In the present study, we induced three kinds of MNGCs: TRAP-positive and CTSK-positive osteoclasts, TRAP-negative and CTSK-positive FBGCs, and TRAPand CTSK-negative FBGCs in the subcutaneous tissue of rats. TRAP-positive osteoclasts, which appeared beside 

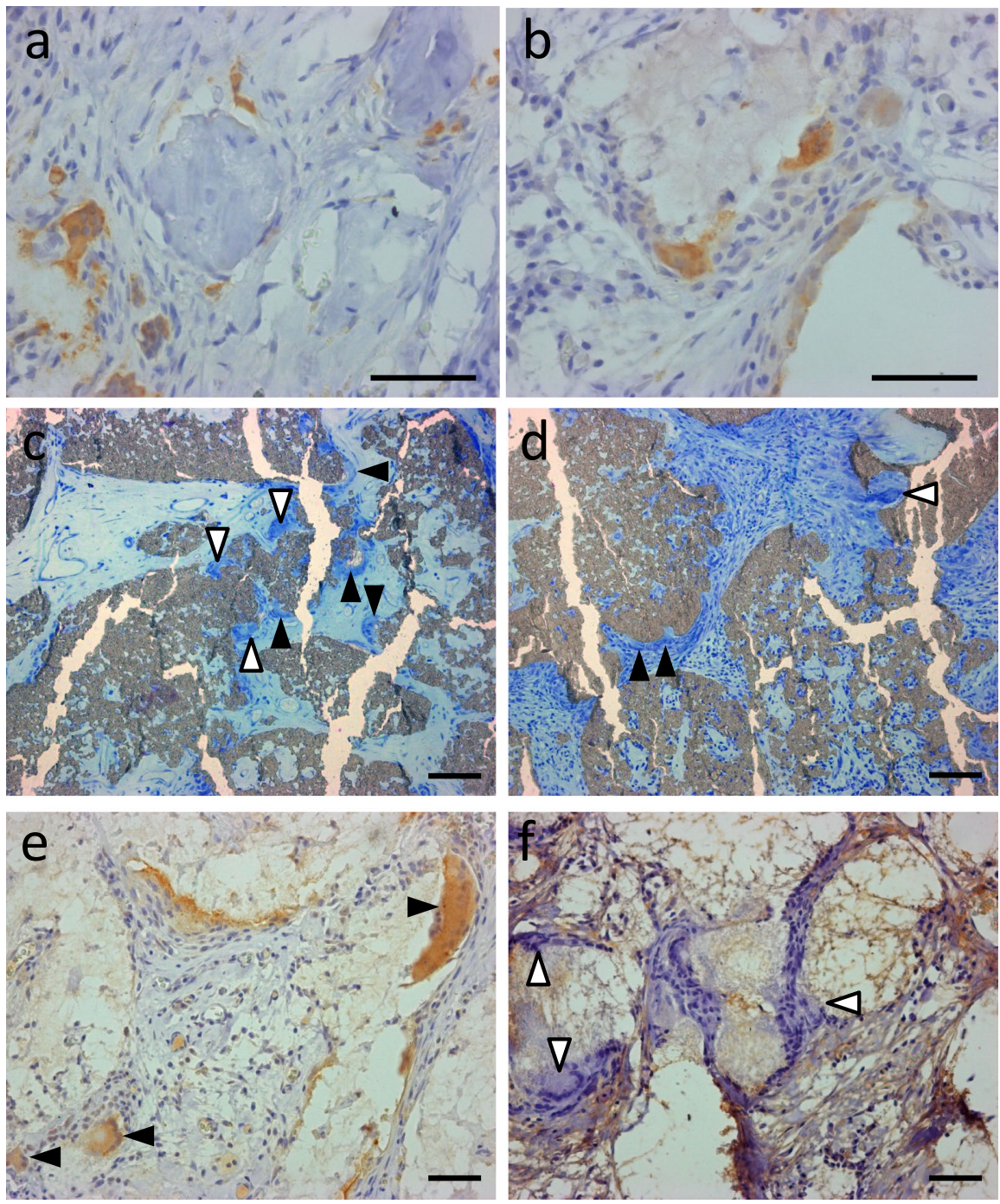

g

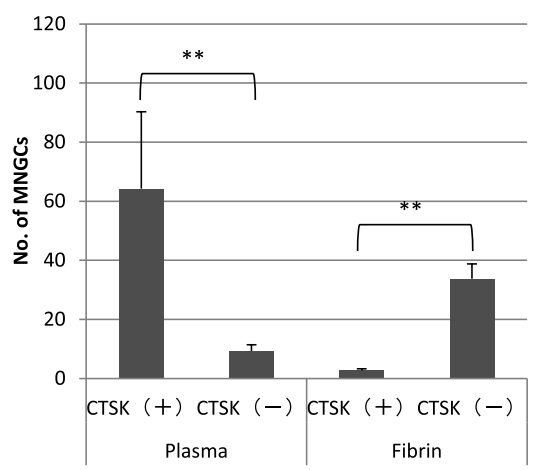

Fig. 7. Immunohistochemical detection of CTSK. (a, b) A specimen implanted with $\beta$-TCP granules with BMMCs accompanied by bone formation (a) and without bone formation (b). (c-f) Specimens implanted with $\beta$-TCP granules only. (c, d) Histological views of specimens stained with Giemsa solution, which were bound with plasma (c) and fibrin (d). White arrowheads and black arrowheads represent FBGCs on the surface of $\beta$-TCP with or without resorption lacunas, respectively. (e, f) Immunohistochemical detection of CTSK in a specimen implanted with $\beta$-TCP granules only, which were bound with plasma (e), and in a specimen implanted with $\beta$-TCP granules were bound with fibrin (f). Black arrowheads indicate FBGCs with a CTSK signal. White arrowheads indicate FBGCs without or with a very faint CTSK signal. Bar $=50 \mu \mathrm{m}$. (g) Quantitation of CTSK-positive and CTSKnegative MNGCs in 3 optical fields at $\times 100$ magnification of specimens implanted with $\beta$-TCP granules bound with plasma and fibrin. $* * P<0.01$. 

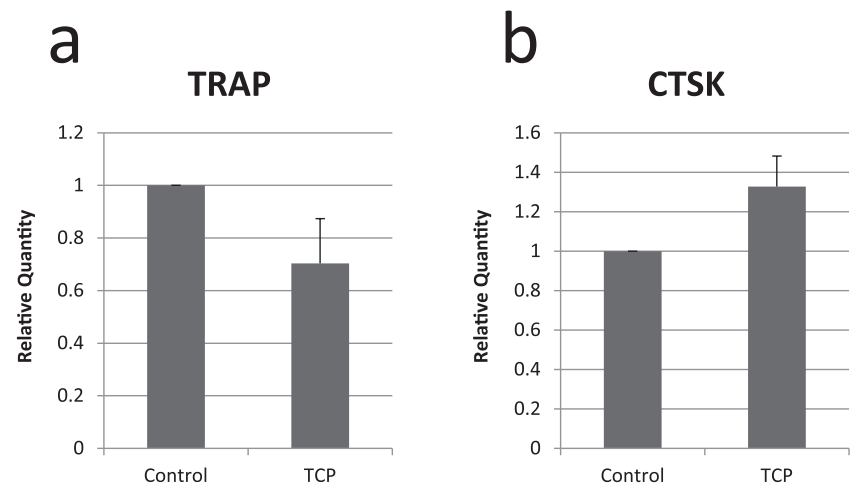

\section{C}

\section{Car2}

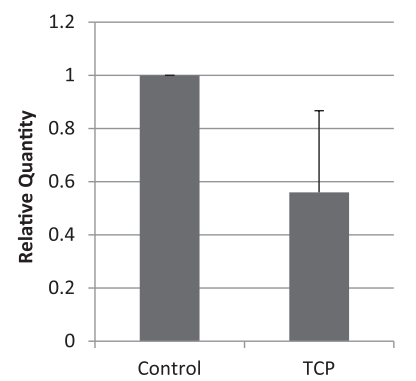

\section{NFATC1}

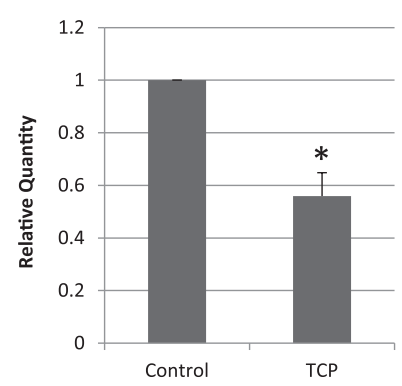

Fig. 8. Real-time PCR analysis for the relative mRNA expression of osteoclast markers in macrophages cultured for 6 days on $\beta$-TCP discs (TCP) or plastic dishes (Control). $* P<0.05$.

bone, markedly degraded $\beta$-TCP granules in these regions, and our results confirmed previous findings showing that bone is essential for osteoclastogenesis [22, 23].

A recent in vitro study suggested that MNGCs induced by the stimulation of IL-4 exhibited transient TRAP activity [12]. Furthermore, FBGCs in several granulomatous lesions were previously found to be positive for TRAP and CTSK using an immunohistochemical procedure [27]. However, the results of our in vivo study strongly suggest that TRAP is a reliable marker of osteoclasts, and this was consistent with previous findings showing that the major osteoclast markers were negative in FBGCs [20].

We identified TRAP-negative and CTSK-positive FBGCs in addition to double negative FBGCs. FBGCs have not been characterized in detail, and the biological activity and significance of these FBGCs, which are more similar to osteoclasts than typical double negative FBGCs, have yet to be clarified. The most important difference between osteoclasts and FBGCs appears to be bone resorbing activity. A previous study reported that the in vitro treatment of macrophages with TNF- $\alpha$ and M-CSF induced osteoclasts [14]. However, these TNF- $\alpha$-induced osteoclasts did not exhibit bone resorbing activity [13, 14]. TNF$\alpha$-induced osteoclasts were also shown to exhibit bone resorbing activity in concert with RANKL $[5,11,17,32$, 34]. These findings strongly suggest that certain similarities

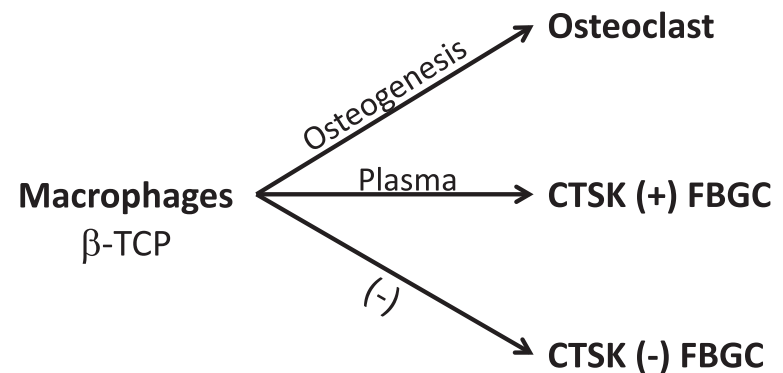

Fig. 9. Factors associated with the formation of the three kinds of MNGCs detected around $\beta$-TCP granules implanted into rat subcutaneous tissue. Osteoclasts were induced in the region with osteogenesis after the implantation of BMMCs. In the presence of plasma, the expression of CTSK was stimulated and CTSK (+) MNGCs were frequently detected. Without plasma, CTSK (-) MNGCs, which had the typical characteristics of FBGCs, were detected.

exist among osteoclasts and FBGCs, and that functional distinctions between osteoclasts and FBGCs may not be as rigid as initially considered.

In contrast to HA, $\beta$-TCP has a biodegradable nature and the results of this study also revealed biodegradability, particularly in the region accompanied by bone formation. Furthermore, we detected the degradation of $\beta$-TCP granules in the region without osteogenesis. TRAP-positive MNGCs were scarcely found in the region without osteogenesis, and $\beta$-TCP was suggested to be degraded by TRAP-negative and CTSK-positive as well as TRAPnegative and CTSK-negative FBGCs. These results suggest functional similarities between FBGCs and osteoclasts. A recent study using an in vitro culture system showed that FBGCs dissolved HA, which was coated on plastic plates, but did not have the ability to resolve bone [30]. Our results suggest that FBGCs dissolve inorganic ceramics and confirmed the findings of the in vitro study.

Based on the results of the present study, we consider the induction of TRAP to be associated with osteogenesis and that of CTSK to be associated with plasma (Fig. 9). $\beta$-TCP itself may also influence the characteristics of MNGCs. Bone marrow macrophages cultured on $\beta$-TCP discs significantly reduced the expression of NFATc1, the key transcriptional factor for the differentiation of osteoclasts, but did not affect the expression of TRAP, CTSK, or Car2. These results suggest that $\beta$-TCP has the potential to interfere with osteoclastogenesis and may contribute to the induction of FBGCs. These results also indicate that CTSK in TRAP-negative FBGCs are induced by a mechanism independent of osteoclast differentiation or direct stimulation by $\beta$-TCP. CTSK is one of the enzymes that digest bone matrix proteins such as collagen, while Car2 has been identified as one of the key enzymes that generate acid in osteoclasts and contribute to the dissolution of bone mineral components. Based on the results of the in vivo analysis, the capacity of FBGCs to dissolve $\beta$-TCP may be one of their general characteristics, and FBGCs may also have the capacity to resorb bone. More detailed studies are 
needed in order to identify CTSK-inducing factors and functional differences between CTSK-positive and CTSKnegative FBGCs. However, the results of the present study suggest that sufficient bleeding effectively induces CTSKpositive FBGCs, which may be advantageous for the replacement of $\beta$-TCP by bone. In conclusion, the implantation of BMMCs with $\beta$-TCP granules was expected to stimulate osteogenesis and the replacement of $\beta$-TCP to bone and may contribute to the regeneration of bone defects. In addition, FBGCs dissolved $\beta$-TCP granules, and biological similarities between MNGCs and osteoclasts have been suggested. Two kinds of FBGCs: CTSK-positive and CTSK-negative FBGCs, were detected in the present study, and certain serum factors were considered to play roles in the induction of CTSK-positive FBGCs.

\section{Acknowledgments}

We thank Prof. Koji Ioku in Keio University for his generous instructions regarding the synthesis of ceramic granules. This work was funded by JSPS KAKENHI 25670834 (to T. I.).

\section{References}

1. Anderson, J. M., Rodriguez, A. and Chang, D. T. (2008) Foreign body reaction to biomaterials. Semin. Immunol. 20; 86-100.

2. Boyle, W. J., Simonet, W. S. and Lacey, D. L. (2003) Osteoclast differentiation and activation. Nature 423; 337-342.

3. Brodbeck, W. G. and Anderson, J. M. (2009) Giant cell formation and function. Curr. Opin. Hematol. 16; 53-57.

4. DeFife, K. M., Jenney, C. R., McNally, A. K., Colton, E. and Anderson, J. M. (1997) Interleukin-13 induces human monocyte/ macrophage fusion and macrophage mannose receptor expression. J. Immunol. 158; 3385-3390.

5. Fuller, K., Murphy, C., Kirstein, B., Fox, S. W. and Chambers, T. J. (2002) TNF alpha potently activates osteoclasts, through a direct action independent of and strongly synergistic with RANKL. Endocrinology 143; 1108-1118.

6. Gonda, Y., Ioku, K., Okuda, T., Kawachi, G., Yonezawa, I., Kurosawa, H. and Ikeda, T. (2008) Application of newly developed globular-shaped granules of beta-tricalcium phosphate for bone substitute. Key Eng. Mater. 361-363; 1013-1016.

7. Gonda, Y., Ioku, K., Shibata, Y., Okuda, T., Kawachi, G., Kamitakahara, M., Murayama, H., Hideshima, K., Kamihira, S., Yonezawa, I., Kurosawa, H. and Ikeda, T. (2009) Stimulatory effect of hydrothermally synthesized biodegradable hydroxyapatite granules on osteogenesis and direct association with osteoclasts. Biomaterials 30; 4390-4400.

8. Ikeda, T., Kasai, M., Suzuki, J., Kuroyama, H., Seki, S., Utsuyama, M. and Hirokawa, K. (2003) Multimerization of the receptor activator of nuclear factor-kappaB ligand (RANKL) isoforms and regulation of osteoclastogenesis. J. Biol. Chem. 278; 47217-47222.

9. Ikeda, T., Gonda, Y., Tatsukawa, E., Shibata, Y., Kamitakahara, M., Okuda, T., Yonezawa, I., Kurosawa, H. and Ioku, K. (2012) Stimulation of osteogenesis in bone defects implanted with biodegradable hydroxyapatite composed of rod-shaped particles under mechanical unloading. Acta Histochem. Cytochem. 45; 283-292.

10. Ikeda, T., Kasai, M., Tatsukawa, E., Kamitakahara, M., Shibata,
Y., Yokoi, T., Nemoto, T. K. and Ioku, K. (2014) A bone substitute with high affinity for vitamin D-binding proteinrelationship with niche of osteoclasts. J. Cell. Mol. Med. 18; 170-180.

11. Jules, J., Shi, Z., Liu, J., Xu, D., Wang, S. and Feng, X. (2010) Receptor activator of NF-\{kappa\}B (RANK) cytoplasmic IVVY535-538 motif plays an essential role in tumor necrosis factor-\{alpha\} (TNF)-mediated osteoclastogenesis. J. Biol. Chem. 285; 37427-37435.

12. Khan, U. A., Hashimi, S. M., Bakr, M. M., Forwood, M. R. and Morrison, N. A. (2013) Foreign body giant cells and osteoclasts are TRAP positive, have podosome-belts and both require OCSTAMP for cell fusion. J. Cell. Biochem. 114; 1772-1778.

13. Kim, N., Kadono, Y., Takami, M., Lee, J., Lee, S. H., Okada, F., Kim, J. H., Kobayashi, T., Odgren, P. R., Nakano, H., Yeh, W. C., Lee, S. K., Lorenzo, J. A. and Choi, Y. (2005) Osteoclast differentiation independent of the TRANCE-RANK-TRAF6 axis. J. Exp. Med. 202; 589-595.

14. Kobayashi, K., Takahashi, N., Jimi, E., Udagawa, N., Takami, M., Kotake, S., Nakagawa, N., Kinosaki, M., Yamaguchi, K., Shima, N., Yasuda, H., Morinaga, T., Higashio, K., Martin, T. J. and Suda, T. (2000) Tumor necrosis factor alpha stimulates osteoclast differentiation by a mechanism independent of the ODF/RANKL-RANK interaction. J. Exp. Med. 191; 275-286.

15. Kzhyshkowska, J., Gudima, A., Riabov, V., Dollinger, C., Lavalle, P. and Vrana, N. E. (2015) Macrophage responses to implants: prospects for personalized medicine. J. Leukoc. Biol. 98; 953-962.

16. Lacey, D. L., Timms, E., Tan, H. L., Kelley, M. J., Dunstan, C. R., Burgess, T., Elliott, R., Colombero, A., Elliott, G., Scully, S., Hsu, H., Sullivan, J., Hawkins, N., Davy, E., Capparelli, C., Eli, A., Qian, Y. X., Kaufman, S., Sarosi, I., Shalhoub, V., Senaldi, G., Guo, J., Delaney, J. and Boyle, W. J. (1998) Osteoprotegerin ligand is a cytokine that regulates osteoclast differentiation and activation. Cell 93; 165-176.

17. Lam, J., Takeshita, S., Barker, J. E., Kanagawa, O., Ross, F. P. and Teitelbaum, S. L. (2000) TNF-alpha induces osteoclastogenesis by direct stimulation of macrophages exposed to permissive levels of RANK ligand. J. Clin. Invest. 106; 14811488 .

18. Matsubara, T., Suardita, K., Ishii, M., Sugiyama, M., Igarashi, A., Oda, R., Nishimura, M., Saito, M., Nakagawa, K., Yamanaka, K., Miyazaki, K., Shimizu, M., Bhawal, U. K., Tsuji, K., Nakamura, K. and Kato, Y. (2005) Alveolar bone marrow as a cell source for regenerative medicine: differences between alveolar and iliac bone marrow stromal cells. J. Bone Miner. Res. 20; 399-409.

19. McNally, A. K. and Anderson, J. M. (1995) Interleukin-4 induces foreign body giant cells from human monocytes/macrophages. Differential lymphokine regulation of macrophage fusion leads to morphological variants of multinucleated giant cells. Am. J. Pathol. 147; 1487-1499.

20. McNally, A. K. and Anderson, J. M. (2011) Foreign body-type multinucleated giant cells induced by interleukin-4 express select lymphocyte co-stimulatory molecules and are phenotypically distinct from osteoclasts and dendritic cells. Exp. Mol. Pathol. 91; 673-681.

21. Moreno, J. L., Mikhailenko, I., Tondravi, M. M. and Keegan, A. D. (2007) IL-4 promotes the formation of multinucleated giant cells from macrophage precursors by a STAT6-dependent, homotypic mechanism: contribution of E-cadherin. J. Leukoc. Biol. 82; 1542-1553.

22. Muto, A., Mizoguchi, T., Udagawa, N., Ito, S., Kawahara, I., Abiko, Y., Arai, A., Harada, S., Kobayashi, Y., Nakamichi, Y., Penninger, J. M., Noguchi, T. and Takahashi, N. (2011) Lineagecommitted osteoclast precursors circulate in blood and settle 
down into bone. J. Bone Miner. Res. 26; 2978-2990.

23. Nakamichi, Y., Mizoguchi, T., Arai, A., Kobayashi, Y., Sato, M., Penninger, J. M., Yasuda, H., Kato, S., DeLuca, H. F., Suda, T., Udagawa, N. and Takahashi, N. (2012) Spleen serves as a reservoir of osteoclast precursors through vitamin D-induced IL-34 expression in osteopetrotic op/op mice. Proc. Natl. Acad. Sci. US A 109; 10006-10011.

24. Ohgushi, H., Okumura, M., Tamai, S., Shors, E. C. and Caplan, A. I. (1990) Marrow cell induced osteogenesis in porous hydroxyapatite and tricalcium phosphate: a comparative histomorphometric study of ectopic bone formation. J. Biomed. Mater. Res. 24; 1563-1570.

25. Okuda, T., Ioku, K., Yonezawa, I., Minagi, H., Kawachi, G., Gonda, Y., Murayama, H., Shibata, Y., Minami, S., Kamihira, S., Kurosawa, H. and Ikeda, T. (2007) The effect of the microstructure of beta-tricalcium phosphate on the metabolism of subsequently formed bone tissue. Biomaterials 28; 2612-2621.

26. Okuda, T., Ioku, K., Yonezawa, I., Minagi, H., Gonda, Y., Kawachi, G., Kamitakahara, M., Shibata, Y., Murayama, H., Kurosawa, H. and Ikeda, T. (2008) The slow resorption with replacement by bone of a hydrothermally synthesized pure calcium-deficient hydroxyapatite. Biomaterials 29; 2719-2728.

27. Park, J. K., Rosen, A., Saffitz, J. E., Asimaki, A., Litovsky, S. H., Mackey-Bojack, S. M. and Halushka, M. K. (2013) Expression of cathepsin $\mathrm{K}$ and tartrate-resistant acid phosphatase is not confined to osteoclasts but is a general feature of multinucleated giant cells: systematic analysis. Rheumatology 52; 1529-1533.

28. Suda, T., Takahashi, N., Udagawa, N., Jimi, E., Gillespie, M. T. and Martin, T. J. (1999) Modulation of osteoclast differentiation and function by the new members of the tumor necrosis factor receptor and ligand families. Endocr. Rev. 20; 345-357.

29. Tatsukawa, E., Gonda, Y., Kamitakahara, M., Matsuura, M., Ushijima, M., Shibata, Y., Yonezawa, I., Fujiwara, M., Ioku, K. and Ikeda, T. (2014) Promotion of normal healing of bone defects under estrogen deficiency by implantation of betatricalcium phosphate composed of rod-shaped particles. $J$. Orthop. Res. 32; 189-196.

30. Ten Harkel, B., Schoenmaker, T., Picavet, D. I., Davison, N. L., de Vries, T. J. and Everts, V. (2015) The foreign body giant cell cannot resorb bone, but dissolves hydroxyapatite like osteoclasts. PLoS One 10; e0139564.

31. Xing, L., Schwarz, E. M. and Boyce, B. F. (2005) Osteoclast precursors, RANKL/RANK, and immunology. Immunol. Rev. $208 ; 19-29$.

32. Yamashita, Y., Ukai, T., Nakamura, H., Yoshinaga, Y., Kobayashi, H., Takamori, Y., Noguchi, S., Yoshimura, A. and Hara, Y. (2015) RANKL pretreatment plays an important role in the differentiation of pit-forming osteoclasts induced by TNFalpha on murine bone marrow macrophages. Arch. Oral Biol. 60; 1273-1282.

33. Yasuda, H., Shima, N., Nakagawa, N., Yamaguchi, K., Kinosaki, M., Mochizuki, S., Tomoyasu, A., Yano, K., Goto, M., Murakami, A., Tsuda, E., Morinaga, T., Higashio, K., Udagawa, N., Takahashi, N. and Suda, T. (1998) Osteoclast differentiation factor is a ligand for osteoprotegerin/osteoclastogenesisinhibitory factor and is identical to TRANCE/RANKL. Proc. Natl. Acad. Sci. U S A 95; 3597-3602.

34. Zhang, Y. H., Heulsmann, A., Tondravi, M. M., Mukherjee, A. and Abu-Amer, Y. (2001) Tumor necrosis factor-alpha (TNF) stimulates RANKL-induced osteoclastogenesis via coupling of TNF type 1 receptor and RANK signaling pathways. J. Biol. Chem. 276; 563-568.

This is an open access article distributed under the Creative Commons Attribution License, which permits unrestricted use, distribution, and reproduction in any medium, provided the original work is properly cited. 